\title{
Increased apoptotic peripheral blood neutrophils in systemic lupus erythematosus: relations with disease activity, antibodies to double stranded DNA, and neutropenia
}

\author{
P A Courtney, A D Crockard, K Williamson, A E Irvine, R J Kennedy, A L Bell
}

\begin{abstract}
Objective-To quantify the percentage of apoptotic peripheral blood neutrophils in systemic lupus erythematosus (SLE) and to determine the relations with disease activity and neutropenia.

Methods-Neutrophil apoptosis in SLE patients $(n=50)$ was assessed by flow cytometry using annexin $\mathrm{V}$ binding and fluorescent labelled anti-fas. Rheumatoid arthritis $(R A, n=20)$ and inflammatory bowel disease patients (IBD, $n=20)$ were studied as disease controls.

Results-The percentage of apoptotic neutrophils, determined by annexin $V$ binding, was increased in peripheral blood of SLE patients (median $=3.25 \%$ ) compared with normal healthy donors ( $n$ $=20$, median $=1.20 \%$ ) and disease controls (RA: median $=1.15 \%)$ (IBD: median $=$ $1.15 \%$ ). SLE neutrophil apoptosis correlated positively with lupus disease activity measured by SLAM score. SLE patients with increased antibodies to dsDNA $(>10$ $\mathrm{mg} / \mathrm{ml}$ ) had increased apoptotic neutrophils. Eight of 14 neutropenic SLE patients had increased apoptotic neutrophils. Increased neutrophil fas expression compared with normal controls was observed in SLE, RA, and IBD.

Conclusion-Neutrophil fas expression is increased non-specifically in inflammatory disease. Increased circulating apoptotic neutrophils in SLE correlate positively with disease activity (SLAM) and may contribute to autoantigen excess including dsDNA.

(Ann Rheum Dis 1999;58:309-314)
\end{abstract}

Systemic lupus erythematosus (SLE) is an autoimmune rheumatic disease characterised by diverse clinical features and the presence of autoantibodies in the serum. The pathogenesis remains unknown but exposure to an environmental trigger, in association with a defined genetic background, may lead to profound changes in the immune system. There is increasing evidence that these changes include defective phagocytosis mechanisms ${ }^{12}$ and increased apoptotic leucocytes. $^{3}$

Apoptosis is a carefully governed process that brings about cell death in a regulated physiological context without the release of inflammatory mediators. The morphological changes of apoptosis are defined ${ }^{4}$ and include contraction of chromatin with nuclear and cytoplasmic budding occurring to form membrane bound fragments or apoptotic bodies. The autoantigens of human SLE, (Ro, La, dsDNA, RNP, etc) are exposed on the surface of apoptotic keratinocytes as a result of this sequence of events. ${ }^{5}$ Therefore, increased apoptotic cells could contribute to autoantigen excess in SLE. ${ }^{6}$

Early during the process of apoptosis leucocytes lose their phospholipid membrane asymmetry that leads to externalisation of phosphatidylserine. ${ }^{7}$ This membrane change is one of the mechanisms by which phagocytic cells identify apoptotic cells. ${ }^{8}$ Experimental evidence indicates that membrane asymmetry detected by annexin $\mathrm{V}$ is a very early and specific phenomenon during apoptosis, ${ }^{9}$ occurring before the typical morphological changes are apparent. Phosphatidylserine can be detected using annexin $\mathrm{V}$, which is a calcium dependent phospholipid binding protein with high affinity for phosphatidylserine. When annexin $\mathrm{V}$ is conjugated to a fluorochrome such as fluorescein isothiocyanate the number of apoptotic cells can be enumerated by flow cytometry. ${ }^{7}$ Increased apoptotic lymphocytes have been reported in $\mathrm{SLE}^{3}$ by this method and it has been suggested that this may be caused by defective clearance by macrophages. ${ }^{12}$

Neutrophil apoptosis is mediated by fas $(\mathrm{APO}-1 / \mathrm{CD} 95)^{10}$ and fas ligation is associated with widespread phosphatidylserine expression on the cell surface. ${ }^{11}$ The fas gene was first described in MRL mice with a lymphoproliferative syndrome including features resembling human SLE. ${ }^{12}$ The human fas gene counterpart (APO-1/CD95) is located on the long arm of chromosome 10 and encodes a transmembrane receptor that, after fas ligand binding actively mediates apoptosis via its cytoplasmic domain. This discovery stimulated the study of apoptosis regulation, but structural abnormalities of apoptotic genes have not been identified to date in human SLE, ${ }^{13}$ although they do occur in rare familial disorders. ${ }^{14}$ In human SLE increased lymphocyte fas expression has been reported but it may not be disease specific. ${ }^{15}$ Fas expression has not previously been studied on SLE neutrophils.

Lupus associated neutropenia (neutrophil count below 1.8 per $\mathrm{n} / \mathrm{l}$, in the absence of cytotoxic drugs) has been reported in at least $20 \%$ of SLE patients ${ }^{16}$ and neutrophil apoptosis has not previously been studied as a potential contributing factor. Given the role of colony stimulating factors in promoting survival of 

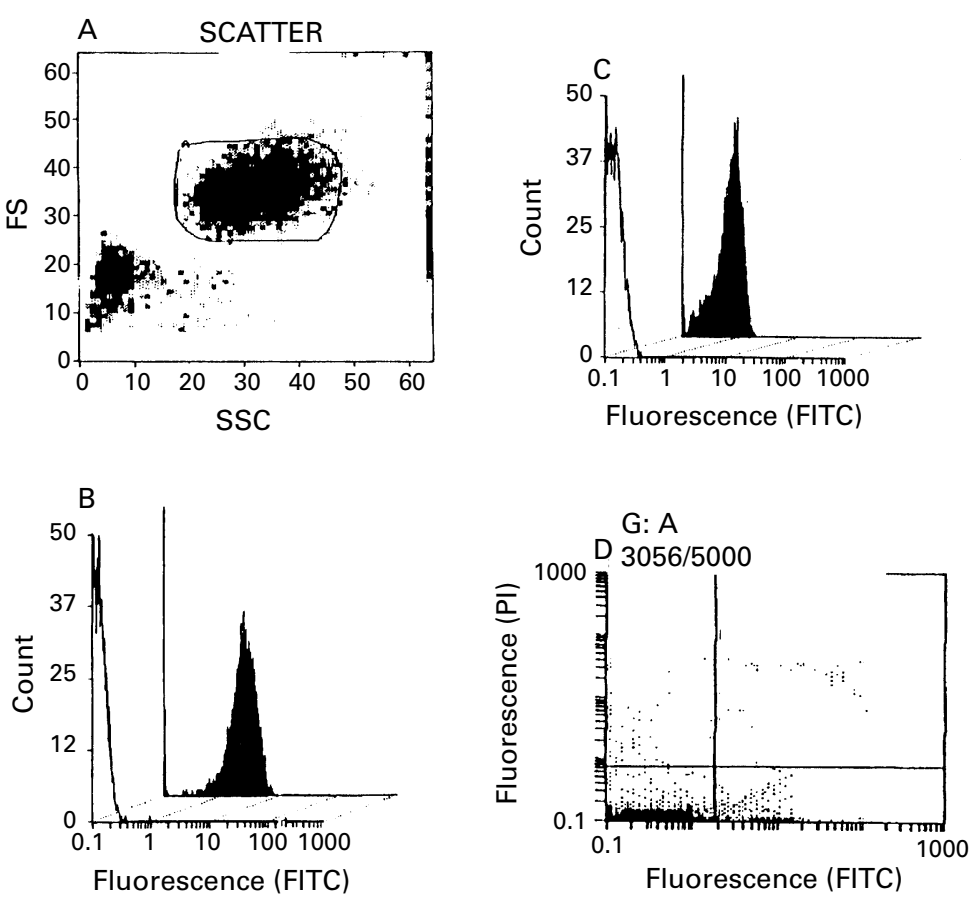

Figure 1 Flow cytometric histograms showing: (A) separated neutrophils from an SLE patient defined by forward angle (FS) and side scatter (SSC) light properties. (B) CD15 positivity (96\%) (shaded area) of the gated neutrophil population and isotype control profile (white area). (C) Fas positivity (68\%) (shaded area) of the gated neutrophil population and isotype control profile (white area). (D) FITC Annexin V and propidium iodide staining of the gated neutrophil population demonstrating $12 \%$ apoptotic cells (quadrant 4; FITC ${ }^{+}, P I^{-}$).
BILAG (British Isles Lupus Activity Guide). These indices have been validated and possess psychometric properties appropriate for clinical research. ${ }^{18}$ The SLAM index covers symptoms that occurred during the previous month and includes 24 clinical and eight laboratory parameters to evaluate organs that cannot otherwise be assessed. The BILAG scale consists of eight symptom based groups and additional laboratory parameters. Previous organ involvement is included in the BILAG score. Drug treatment and prednisolone dose were recorded for all patients. Serum antibodies to dsDNA were measured by a commercial assay using the Farr technique (Immunodiagnostics). Rheumatoid arthritis disease activity was assessed by erythrocyte sedimentation rate (ESR), C reactive protein (CRP), and Ritchie articular index. IBD activity was assessed by ESR and CRP.

CELL PREPARATION

Peripheral blood was sampled and layered on equal volumes of Histopaque 1119 and Histopaque 1077 (Sigma) to obtain an enriched granulocyte layer. ${ }^{19}$ Cells were washed in PBS and counted before labelling.

\section{NEUTROPHIL MORPHOLOGY}

Cytocentrifuge slides were prepared $(n=10)$ within four hours of venepuncture to confirm the presence of neutrophils with morphological features of apoptosis.

Slides were stained with modified Wright

neutrophils by suppressing apoptosis, in addition to granulopoietic properties, the interactions of these factors have also been studied.

We present data on fas expression and numbers of apoptotic neutrophils in SLE. Disease activity and drug treatment have been recorded and correlated with data about apoptosis. In addition to studying normal control samples, we have measured a rheumatic chronic inflammatory disease control (rheumatoid arthritis) and a non-rheumatic chronic inflammatory disease control group (inflammatory bowel disease) in an attempt to discover the specificity of changes seen.

\section{Methods}

PATIENTS AND CONTROLS

All SLE patients $(n=50)$ fulfilled ARA criteria for diagnosis ${ }^{17}$ and were recruited from outpatient clinics and rheumatology inpatient ward. Disease controls, rheumatoid arthritis (RA) $(n=20)$ and inflammatory bowel disease (IBD) $(n=20)$ patients were recruited from rheumatology and surgical outpatients respectively and inpatient wards. Patients with disease flare ups were studied before the institution of additional drug treatment. SLE patients taking cytotoxic drugs were excluded from the study. Written informed consent was obtained from all patients. Age and sex matched normal healthy volunteers were recruited from hospital staff.

\section{CLINICAL INFORMATION}

Clinical features were recorded after full clinical examination and lupus patients were assigned precise diagnostic subgroups where appropriate. SLE disease activity was assessed by SLAM (Systemic Lupus Activity Measure) and stain and apoptotic neutrophils counted under high power view in 500 cells.

\section{IMMUNOFLUORESCENCE LABELLING}

Fas, CD15: Aliquots of $100 \mu \mathrm{l}$ cell suspension $\left(5 \times 10^{6}\right.$ cells $\left./ \mathrm{ml}\right)$ were incubated for 60 minutes at $4^{\circ} \mathrm{C}$ with $10 \mu \mathrm{l}$ anti-fas-FITC (Immunotech), $10 \mu \mathrm{l}$ MsIgG1-FITC (Immunotech) or $20 \mu \mathrm{l}$ anti-CD15-PE (Coulter) respectively. MsIgG1 was used as an isotype control for anti-fas to allow subtraction of non-specific staining. After two washes in phosphate buffered saline (PBS) the cells were resuspended in PBS before flow cytometric analysis.

Annexin V: Aliquots of $100 \mu \mathrm{l}$ cells $\left(5 \times 10^{6}\right.$ cells $/ \mathrm{ml}$ ) were suspended in binding buffer ( $\mathrm{R}$ and D) and incubated for 20 minutes with annexin V-FITC ( $\mathrm{R}$ and $\mathrm{D}$ ) and propidium iodide ( $\mathrm{R}$ and $\mathrm{D}$ ). Immediate flow cytometric analysis was performed.

\section{FLOW CYTOMETRY}

Flow cytometric analyses were performed on a Coulter EPICS ELITE flow cytometer that was standardised for inter-and intra-run variability by calibration with Immunocheck fluorospheres (Coulter). Neutrophils were identified on the basis of light scatter properties and CD15 positivity (>95\%). Five thousand neutrophils were analysed per sample and the percentage of fas positive cells determined after subtraction of non-specific staining as identified by the isotype control histogram.

Dual colour histograms were analysed for annexin V/propidium iodide labelled cells and the percentages of apoptotic $\left(\mathrm{FITC}^{+} \mathrm{PI}^{-}\right)$and necrotic $\left(\mathrm{FITC}^{+} \mathrm{PI}^{+}\right.$) cells determined (fig 1). 


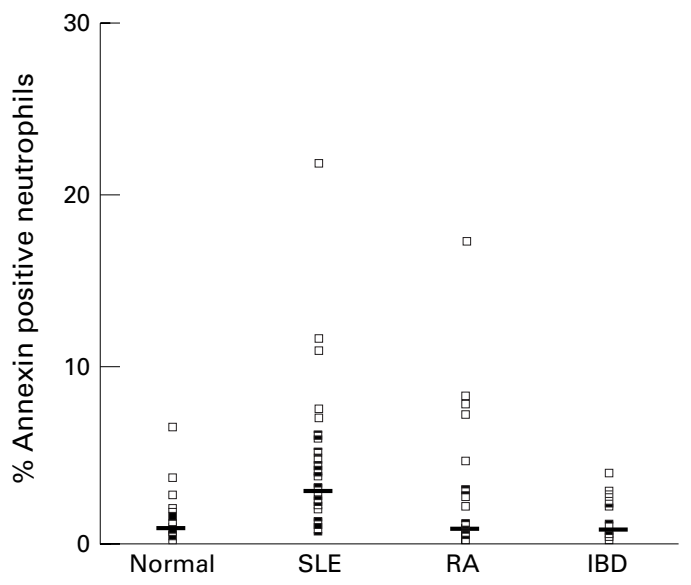

Figure 2 Percentage annexin $V$ positive neutrophils in normal controls, SLE patients, $R A$ patients and IBD patients. Each point represents one patient, and the median for each group is shown.

DETERMINATION OF SERUM GRANULOCYTE COLONY STIMULATING FACTOR (G-CSF) AND GRANULOCYTE MACROPHAGE COLONY STIMULATING FACTOR (GM-CSF)

Serum G-CSF and GM-CSF were measured by ELISA (Quantikine Immunoassay) according to the manufacturer's instructions.

STATISTICAL ANALYSIS

The statistical significance of the results was determined using the Mann-Whitney test for unpaired samples. Spearman correlation coefficients were used to study the relations between disease activity or treatment and apoptosis measures.

\section{Results}

ANNEXIN $v$

There was a significant increase of apoptotic neutrophils (fig 2) as assessed by the annexin V assay in SLE patients $(n=50$, median $=3.25$, interquartile range $2.28-5.23$ ) compared with normal controls $(n=20$, median $=1.20$, interquartile range $0.43-1.88 ; \mathrm{p}=0.001)$, RA patients $(n=20$, median $=1.15$, interquartile range $0.38-4.40 ; \mathrm{p}=0.008)$, and IBD patients $(\mathrm{n}=20$, median $=1.15$, interquartile range $0.10-2.95 ; \mathrm{p}=0.008)$. There was no signifi-

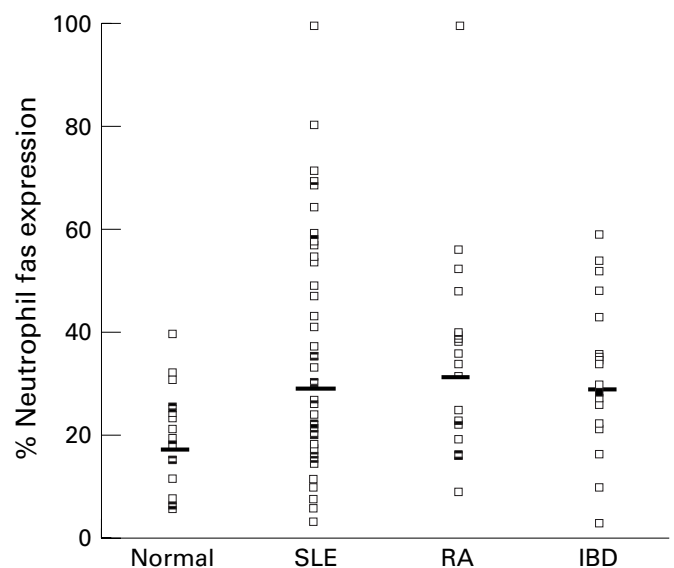

Figure 3 Percentage neutrophil fas expression in normal controls, SLE patients, RA patients, and IBD patients. Each point represents an individual patient and the median for each group is shown.

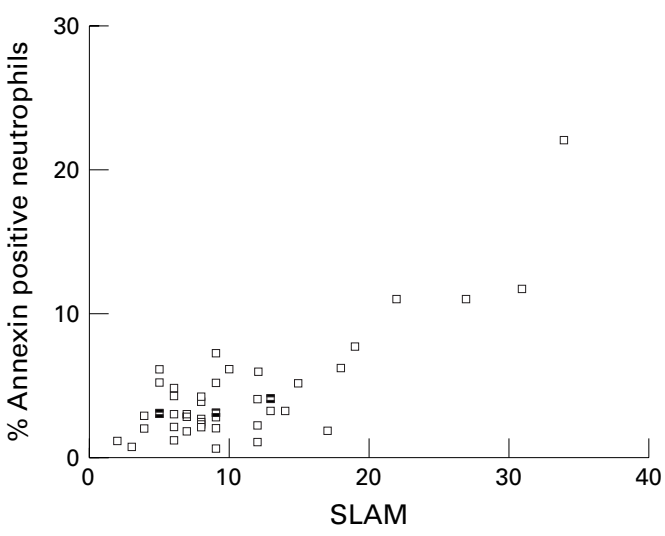

Figure 4 Scatter plot demonstrating positive correlation between percentage annexin $V$ positive neutrophils and SLAM $(r=0.50, p=0.001)$. Each point represents an individual SLE patient.

cant difference in the number of apoptotic neutrophils between the RA $(\mathrm{p}=0.75)$ or IBD $(p=0.68)$ and normal control groups.

NEUTROPHIL MORPHOLOGY

The correlation observed between the percentage of apoptotic neutrophils by morphology $(\mathrm{n}=10)$ and by annexin $\mathrm{V}$ in individual patients was not statistically significant $(r=0.59, \mathrm{p}=0.07$ : Spearman). The morphology data were also assessed in two groups: $(<2 \%$ apoptotic neutrophils, mean $1.4 \%, \mathrm{n}=5)$ and $(>2 \%$ apoptotic neutrophils, mean $2.8 \%, \mathrm{n}=5$ ). The first group $(<2 \%)$ had mean percentage annexin positivity of $2.6 \%$ and the second group ( $>2 \%$ ) had mean percentage annexin positivity of $5.2 \%$ suggesting that patients with increased numbers of apoptotic cells by annexin $\mathrm{V}$ also had increased apoptotic cells by morphology. The percentage of apoptotic neutrophils was higher, in all samples $(\mathrm{n}=10)$ by annexin $\mathrm{V}($ mean $=3.6 \%$ ) than by morphology (mean $=1.7 \%$ ).

FAS

Neutrophil fas expression (fig 3) was significantly increased in SLE (median $=30.1$, IQ range 18.00-53.58; $\mathrm{p}=0.002$ ), $\mathrm{RA}$ (median $=$ 32.85, IQ range 22.18-39.98; $\mathrm{p}=0.001$ ), and $\mathrm{IBD}($ median $=28.95, \mathrm{IQ}$ range $23.00-41.48 ; \mathrm{p}$ $=0.006)$ compared with normal controls (median=17.8, IQ range 8.50-24.93). There was no significant difference between neutrophil fas expression in either RA or IBD when compared with SLE ( $p=0.88$ and $p=0.68$ respectively). There was a positive correlation between SLE neutrophil fas expression and neutrophil annexin positivity $(r=0.52, \mathrm{p}=0.01)$.

\section{DISEASE ACTIVITY}

SLE

At the time of study 10 patients had inactive disease (SLAM <6), 26 had moderately active disease (SLAM 6-12), and 14 had active disease (SLAM >12).

Correlations of SLE apoptotic neutrophil count and fas expression with disease activity measures were determined. There was a positive correlation between annexin $\mathrm{V}$ positive neutrophils and SLAM $(r=0.50, \mathrm{p}=0.001)$ (fig 4 ). When the outlier point in figure 4 was eliminated the Spearman's correlation coeffi- 


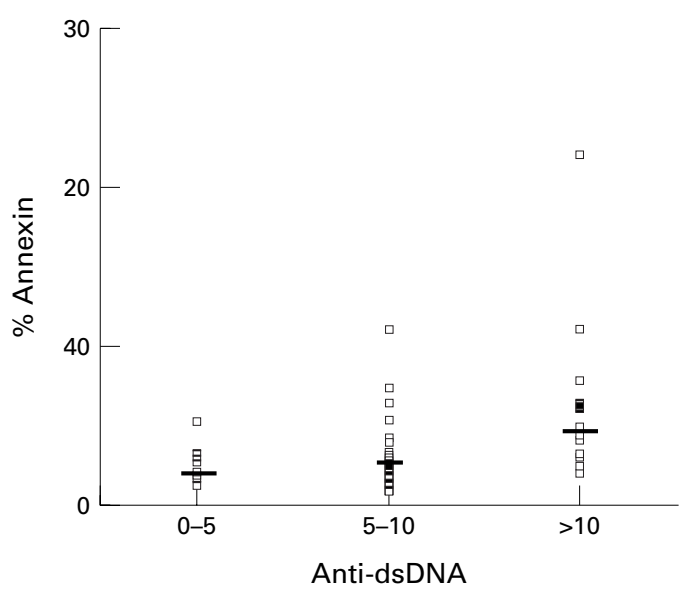

Figure 5 Percentage annexin $V$ expression in SLE patients stratified according to the concentration of antibodies to double stranded DNA $(\mathrm{mg} / \mathrm{ml})$. The 5-10 group includes concentrations greater than 5.0 and less than or equal to 10.0. Each point represents an individual patient and medians are marked for each group.

cient was $0.45, \mathrm{p}=0.002$. There was also a positive correlation between SLE neutrophil fas expression and $\operatorname{ESR}(r=0.54, \mathrm{p}=0.001)$.

$R A$

RA disease activity did not correlate with the annexin $\mathrm{V}$ positive neutrophil count. However, there was a positive correlation between neutrophil fas expression and CRP $(r=0.58$, $\mathrm{p}=0.003)$.

$I B D$

There were no significant correlations between annexin $\mathrm{V}$ positive neutrophils or neutrophil fas expression and disease activity (ESR and CRP).

ANTIBODIES TO DSDNA

There was a positive correlation between serum antibodies to dsDNA and percentage apoptotic neutrophils $(r=0.40, \mathrm{p}=0.007)$. Antibodies to dsDNA (normal range $<5$ $\mathrm{mg} / \mathrm{ml})$ were stratified into three groups: $(0-5$ $\mathrm{mg} / \mathrm{ml}, \mathrm{n}=12),(5-10 \mathrm{mg} / \mathrm{ml}, \mathrm{n}=20)$ and $(>10$ $\mathrm{mg} / \mathrm{ml}, \mathrm{n}=18)$. There were significantly increased apoptotic neutrophils in the $>10$ $\mathrm{mg} / \mathrm{ml}$ dsDNA group (median $=4.9 \%$ ) compared with the $5-10 \mathrm{mg} / \mathrm{ml}$ group (median = $2.9 \%, \mathrm{p}=0.007$ ) and the $0-5 \mathrm{mg} / \mathrm{ml}$ (median $=2.75, \mathrm{p}=0.006$ ) (fig 5).

There was no statistically significant correlation observed between antibodies to dsDNA

Table 1 Annexin, fas, SLAM, and colony stimulating factor data for neutropenic SLE patients $(n=14)$

\begin{tabular}{llllll}
\hline Neutrophil count & \%fas & \%annexin & SLAM & G-CSF (pg/ml) & GM-CSF (pg/ml) \\
\hline $1.08 \times 10^{3} / \mu 1$ & 99.0 & 6.3 & 18 & 291.90 & 2.85 \\
1.80 & 41.2 & 7.8 & 19 & 69.93 & 0.99 \\
1.40 & 69.1 & 11.1 & 27 & 53.4 & 1.47 \\
1.80 & 24.0 & 2.2 & 6 & 53.50 & 1.47 \\
1.30 & 21.8 & 3.0 & 4 & 28.14 & 0.18 \\
1.30 & 5.4 & 2.2 & 8 & 1.00 & 0.60 \\
1.40 & 27.1 & 3.1 & 7 & 28.74 & 0.25 \\
1.80 & 22.2 & - & 15 & 20.21 & 0.92 \\
1.02 & 99.0 & 22.0 & 34 & - & - \\
1.36 & 53.3 & 1.9 & 17 & - & - \\
1.70 & 43.2 & 2.8 & 8 & - & - \\
0.72 & 54.4 & 11.1 & 22 & - & - \\
1.80 & 49.0 & 5.3 & 5 & - & - \\
1.70 & 37.2 & 4.2 & 13 & - & \\
\hline
\end{tabular}

and SLE disease activity measures (SLAM: $r=$ $0.25, \mathrm{p}=0.08)$.

RELATION OF NEUTROPENIA TO G-CSF AND GM-CSF

Circulating G-CSF was increased in four of eight patients with neutropenia. Details of the neutropenic patients are recorded in table 1. G-CSF concentrations were increased in two lupus patients without neutropenia but were within the normal range in other lupus patients $(\mathrm{n}=6)$, RA patients $(\mathrm{n}=4)$ and normal controls $(n=6)$ (data not shown). GM-CSF concentrations were within the normal range $(<7.8 \mathrm{pg} / \mathrm{ml})$ in all samples.

TREATMENT

To analyse the possible effects of treatment on the apoptotic cell counts we correlated the number of apoptotic cells with the corticosteroid dose (range 0-60 mg). Twenty SLE, 13 $\mathrm{RA}$, and 12 IBD patients were taking corticosteroids at the time of sampling.

There was no significant inverse correlation between corticosteroid dose and neutrophil apoptosis $(r=-0.32, \mathrm{p}=0.14)$. When patients were classified as those taking high dose corticosteroids $(>7.5 \mathrm{mg})$, those taking low dose corticosteroids $(<7.5 \mathrm{mg}$ ) and those taking no corticosteroid there was a significant difference in apoptotic neutrophil count between the no corticosteroid group (median 5.3\%) and the high dose corticosteroid group (median = $2.3 \%, \mathrm{p}=0.02$ ). Four patients with active disease including neutropenia were studied before treatment and again six weeks after being established receiving corticosteroid treatment. Reduced disease activity coincided with decreased neutrophil fas expression and decreased apoptotic neutrophil count. However, a large study would be necessary to assess the effects of corticosteroids on neutrophil apoptosis in SLE.

\section{Discussion}

This study shows a significantly higher level of circulating apoptotic neutrophils in SLE compared with normal controls and disease controls (RA and IBD). The experimental design with FITC-annexin V staining of freshly isolated neutrophils was used to detect apoptosis in the circulation and avoid in vitro artefacts. There was not a statistically significant positive correlation between the percentage apoptotic cells identified by morphology and annexin V in individual patients $(r=0.59$, $\mathrm{p}=0.07$ : Spearman) and more cells were recognised by annexinV. This may have been partly because of the fact that annexin $\mathrm{V}$ identifies apoptotic cells at an earlier stage than morphology. Taken with the reported finding of high concentrations of circulating early apoptotic peripheral blood mononuclear cells, ${ }^{3}$ this study is concordant with the suggestion that impaired clearance of apoptotic material may be a specific hallmark of SLE. In addition, or alternatively, increased neutrophil apoptosis may be occurring.

For example positive correlations were observed between apoptotic neutrophil counts 
and SLE disease activity measures. Although increased apoptotic lymphocytes have been reported in $\mathrm{SLE}^{31520}$ it is not clear if there is a correlation with disease activity and one study has reported the presence of apoptotic monocytes in the circulation of patients with active lupus. ${ }^{21}$ The finding of increased numbers of apoptotic neutrophils in the peripheral circulation could be more important as neutrophils are numerically greater and have a reduced life span compared with mononuclear cells. Therefore, increased apoptotic neutrophils could be a greater potential source of lupus specific autoantigens and failure of clearance could trigger or exacerbate SLE. Our demonstation of increased apoptotic neutrophils provides one possible mechanism for the increase in antibodies to ds DNA in SLE. However, increased antibodies to dsDNA are associated with disease flares ${ }^{22}$ and the increased apoptotic neutrophils may not directly result in antibody formation.This study, however, showed a statistically stronger correlation between dsDNA antibodies and apoptotic neutrophils than between dsDNA and disease activity.

Fas expression was also increased in RA and IBD, which suggests that switching on of neutrophil death genes may be a general characteristic of inflammatory disorders designed to contribute to pathways of inflammatory resolution. ${ }^{23}$ Indeed, our group have previously shown that inflammatory synovial fluid contains a factor or factors capable of promoting neutrophil apoptosis. ${ }^{24}$ However, the inhibitory effect of corticosteroids on neutrophil apoptosis does not fit easily with proposed antiinflammatory mechanisms of action. ${ }^{25} 26$ In contrast with the effect on neutrophil apoptosis corticosteroids are inducers of apoptosis in lymphoid cells. ${ }^{27}$

There was a discrepancy between neutrophil fas expression in normal controls between this study and that reported by Iwai et al, who reported that neutrophils expressed fas constitutively. ${ }^{28}$ Fas expression on mononuclear cells was comparable (data not shown) and the differences for neutrophils may reflect the immediate staining and analyses performed in this study as neutrophils rapidly express fas and undergo apoptosis after removal from the circulation.

The discrepancy between annexin V positive neutrophils in SLE and RA requires explanation. The relation between neutrophil fas expression and apoptosis is likely to be influenced by multiple factors and not all fas engagement necessarily results in apoptosis. ${ }^{29}$ The regulation of apoptosis is complex and pathways other than fas can activate machinery for cell death. ${ }^{30}$ In addition, serum soluble fas can protect cells from fas mediated apoptosis, ${ }^{31}$ so additional regulatory input occurs within the fas pathway itself.

Apoptotic cell clearance is now the focus of attention in SLE. ${ }^{32}$ There is evidence of a decreased phagocytic clearance of apoptotic ${ }^{12}$ cells but this will require further evaluation. Savill et $a l^{33}$ have reported in vitro uptake of apoptotic neutrophils by macrophages in nor- mal controls and some cases of inflammatory arthritis to be similar but this method has not yet been applied in SLE. The macrophage receptors involved in the uptake of apoptotic cells are being defined ${ }^{834}$ and this may facilitate investigation of a specific defect in lupus. The clearance of apoptotic cells does not usually cause activation of the immune system and phagocytic cells have enormous capacity to engulf apoptotic material effectively and rapidly. ${ }^{34}$ However, in the situation of impaired phagocytosis or increased apoptosis an overwhelming quantity of antigenic material from apoptotic bodies may lead to a permanent challenge to natural tolerance. The presence of large quantities of nucleosomes ${ }^{36}$ may result in the presentation of lupus specific autoantigens to autoreactive $\mathrm{T}$ cells. In genetically susceptible people this sequence of events may result in polyclonal B lymphocyte stimulation and autoantibody responses typical of SLE, leading to the induction and maintenance of autoimmunity.

SLE associated neutropenia is likely to be multifactorial. Normal or hyperplastic granulopoiesis has been reported in untreated SLE neutropenia; most recently in all of nine patients successfully treated with recombinant G-CSF. ${ }^{37}$ Patients with SLE can also exhibit diminished granulopoiesis and a hypocellular marrow attributable to myelofibrosis or aplasia. ${ }^{38}$ Circulating anti-neutrophil antibodies occur in SLE but their presence in patients with and without neutropenia ${ }^{39}$ suggests they are not the most important factor responsible.

Neutrophil apoptosis has not previously been studied as a potential contributing factor to SLE neutropenia. The suppression of neutrophil apoptosis by effective treatments of SLE neutropenia such as recombinant granulocyte colony stimulating factor ${ }^{40}$ and corticosteroids $^{2526}$ are in keeping with this theory.

Serum G-CSF is increased in other neutropenic disease states ${ }^{41}{ }^{42}$ and this study suggests that G-CSF is increased in some patients with lupus neutropenia but not in others.

The possibility exists that increased neutrophil apoptosis may aggravate some cases of lupus neutropenia associated with active disease. However, the increased apoptotic neutrophils demonstrated in these patients may be a result of disease activity and neutropenia may occur by different mechanisms. No significant conclusion could be reached regarding the relation between neutrophil apoptosis, SLE neutropenia, and G-CSF concentrations from this study.

Corticosteroid treatment was associated with reduced apoptotic neutrophils, which would have been predicted given the findings of in vitro studies. The inhibition of neutrophil apoptosis may represent an important mechanism of action in the treatment of disease flares in SLE.

The finding of increased circulating peripheral blood apoptotic neutrophils that correlates with SLE disease activity keeps the process of apoptosis at the centre of pathogenetic interest in the generation of autoantibodies. The apop- 
tosis related sequelae of experimental treatments are already the focus of attention ${ }^{43}$ and the development of new therapeutic modalities in rheumatic diseases may be orientated towards the biological regulation of cell survival. $^{44}$

Funding: this work was supported by LUPUS UK and a Royal Victoria Hospital, Belfast, Research Fellowship.

The authors thank Dr S A McMillan for his assistance in the measurement of antibodies to dsDNA.

1 Herrmann M, Voll RE, Zoller OM, Hagenhoffer M, Ponne $\mathrm{BB}$, Kalden JR. Impaired phagocytosis of apoptotic cell material by monocyte-derived macrophages from patients with systemic lupus erythematosus. Arthritis Rheum 1998;41:1241-50.

2 Herrmann M, Zoller OM, Hagenhoffer M, Voll R, Kalden JR. What triggers anti-dsDNA antibodies? Mol Biol Rep

3 Perniok A, Wedekind F, Herrmann M, Specker C, Schneider M. High levels of circulating early apoptotic peripheral blood mononuclear cells in SLE. Lupus 1998;7 113-18.

4 Kerr JFR, Wyllie AH, Currie AR. Apoptosis; a basic biological phenomenon with wide ranging implications in tissue kinetics Br J Cancer 1972;26:239-57.

5 Casciola-Rosen LA, Anhalt G, Rosen A. Autoantigens targeted in SLE are clustered in two populations surface structures on apoptotic keratinocytes. J Exp Med 1994: 179:1317-30.

6 Utz PJ, Anderson P. Posttranslational protein modifications, apoptosis, and the bypass of tolerance to autoantigens. Arthritis Rheum 1998;41:1152-60.

7 Koopman G, Reutlingsberger CPM, Kuitjen GAM, Keehnen RMJ, Pals ST, van Oers MHJ. Annexin V for flow nen RMJ, Pals ST, van Oers MHJ. Annexin V for flow cytometric detection of phosphatidylserine expression

8 Fadok VA, Voelker DR, Campbell PA, Cohen JJ, Bratton DL, Henson PM. Exposure of phosphatidylserine on the surface of apoptotic lymphocytes triggers specific recognition and removal by macrophages. J Immunol 1992;148 $2207-16$

9 Engeland M, Ramaekers F, Schutte B, Reutelingsperger CPM. A novel assay to measure loss of plasma membrane asymmetry during apoptosis of adherent cells in culture. Cytometry 1996;24:131-9.

10 Liles WC, Klebanoff SJ. Regulation of apoptosis in neutrophils-Fas track to death? J Immunol 1995;155: 3289-91.

11 Martin SI, Finucane DM, Amarante-Mendes GP, O'Brien GA, Green DR. Phosphatidylserine externalization during ICE/CED-3 protease activity. J Biol Chem 1996;271 28753-6.

12 Watanabe Fukunaga R, Brannan CI, Copeland NG, Jenkin NA, Nagata S. Lymphoproliferation disorder in mice explained by defects in fas antigen that mediates apoptosis. Nature 1992;356:314-17

13 Mysler E, Bina P, Drippa J, Ramos P, Friedman SM, Krimmer $\mathrm{PH}$, et al. The APO-1/fas protein in human systemic lupus erythematosus. J Clin Invest 1994; 93:1029-34.

14 Drappa J, Vaishnaw AK, Sullivan KE, Chu JL, Elkon KB. Fas gene mutations in the Canale-Smith syndrome, an inherited lymphoproliferative disorder associated with autoimmunity. N Engl J Med 1996;335:1643-9.

15 Lorenz HM, Grunke M, Hieronymus T, Herrmann M, Lorenz HM, Grunke $M$, Hieronymus T, Herrmann $M$,
Kuhnel A, Manger B, et al. In vitro apoptosis and expression of apoptosis related molecules in lymphocytes expression of apoptosis related molecules in lymphocytes
from patients with SLE and other autoimmune diseases. from patients with SLE and other

16 Keeling DM, Isenberg DA. Haematological manifestations of systemic lupus erythematosus. Blood Rev 1993;7:199207.

17 Tan EM, Cohen AS, Fries JF, Masi AT, McShane DJ, Rothfield NF, et al. The 1982 revised criteria for the classification of systemic lupus erythematosus. Arthritis Rheum 1982;25:1271-7.

18 Liang MH, Socher A, Larson MG, Schur PH. Reliability and validity of six systems for the clinical assessment of disease activity in SLE. Arthritis Rheum 1989;32:1107-18.

19 English D, Anderson BR. Single step separation of red blood cells, granulocytes and mononuclear cells on discon- tinuous density gradient of ficoll-hypaque. J Immunol Methods 1974;5:249-52.

20 Emlen W, Niebur JA, Adera R. Accelerated in vitro apoptosis of lymphocytes from patients with systemic lupus erythematosus. J Immunol 1994;152:3685-92.

21 Richardson BC, Yung RL, Johnson KJ, Rowse PE, Lalwani ND. Monocyte apoptosis in patients with active lupus. Arthritis Rheum 1996;39:1432-4.

22 Bootsma H, Spronk PE, Ter Borg EJ, Hummel EJ, de Boer G, Limburg PC, et al. The predictive value of fluctuations in IgM and IgG class anti-dsDNA antibodies for relapse in systemic lupus erythematosus. Ann Rheum Dis 1997;56: 6ystemic

23 Savill JS, Haslett C. Granulocyte clearance by apoptosis in the resolution of inflammation. Cell Biol 1995;6:385-93.

24 Bell AL, Magill MK, McKane R, Irvine AE. Human blood and synovial fluid neutrophil cultured invitro undergo programmed cell death which is promoted by the addition of synovial fluid. Ann Rheum Dis 1995;54:910-15.

25 Liles WC, Dale DC, Klebanoff SJ. Glucocorticoids inhibit apoptosis of human neutrophils. Blood 1995;86:3181-8.

26 Cox G. Glucocorticoid treatment inhibits apoptosis in human neutrophils. J Immunol 1995;154:4719-25.

27 Cohen JJ, Duke R. Glucocorticoid activation of a calciumdependent endonuclease in thymocyte nuclei leads to cell death. J Immunol 1984;132:38-42.

28 Iwai K, Miyawaki T, Takizawa T, Konno A, Ohta K, Yachie A, et al. Differential expression of bcl-2 and susceptibility to anti-fas-mediated cell death in peripheral blood lymphocytes, monocytes and neutrophils. Blood 1994;84: 1201-8.

29 Yonehara S, Ishii A, Yonehara M. A cell killing monoclonal antibody (anti-fas) to a cell surface antigen coantibody (anti-fas) to a cell surface antigen coJ Exp Med 1989;169:1747-52.

30 Nagata S. Apoptosis by death factor. Cell 1997;88:355-65.

31 Cheng J, Zhou T, Liu C, Shapiro JP, Brauer MJ, Kiefer MC, et al. Protection from fas mediated apoptosis by a soluble form of the fas molecule. Science 1994;263:1759-62

32 Manfredi AA, Rovere P, Galati G, Heltai S, Bozzolo E, Soldini $\mathrm{L}$, et al. Apoptotic cell clearance in systemic lupus erythematosus. Arthritis Rheum 1998;41:205-14

33 Savill JS, Wyllie AH, Henson JE, Walport MJ, Henson PM, Haslett C. Macrophage phagocytosis of aging neutrophils in inflammation. J Clin Invest 1989;83:865-75.

34 Ren Y, Silverstein CL, Allen J, Savill J. CD36 gene transfer confers capacity for phagocytosis of cells undergoing apoptosis. J Exp Med 1995;181:1857-62.

35 Hart SP, Dougherty GJ, Haslett C, Dransfield I. CD44 regulates phagocytosis of apoptotic neutrophil granulocytes, but not apoptotic lymphocytes by human macrophages. J Immunol 1997;159:919-25.

36 Bell DA, Morrison B. The spontaneous apoptotic death of normal human lymphocytes in vitro; the release of, and immunoproliferative response to, nucleosomes in vitro. Clin Immunol Immunopathol 1991;60:13-26.

37 Euler HH, Harten P, Zeuner RA, Schwab UM. Recombinant granulocyte colony stimulating factor in patients with systemic lupus erythrmatosus associated neutropenia with systemic lupus erythrmatosus associated neutropen

38 Rosenthal NS, Farhi DC. Bone marrow findings in connective tissue disease. Am J Clin Pathol 1989;92:650-4.

39 Minchinton RM, Waters AH. The occurrence and significance of neutrophil antibodies. Br J Haematol 1984;56 521-8.

40 Mempel K, Pietsch T, Menzel T, Zeidler C, Welte K. Increased serum levels of granulocyte colony stimulating
factor in patients with severe congenital neutropenia. Blood 1991;77:1919-22.

41 Brach MA, de Vos S, Gus HJ, Herrmann P. Prolongation of survival of human polymorphonuclear neutrophils by
granulocyte-macrophage colony-stimulating factor is caused by inhibition of programmed cell death. Blood 1992;80:2920-4.

42 Sallerfors B. Endogenous production and peripheral blood levels of granulocyte macrophage and granulocyte colony stimulating factors (GM-CSF and G-CSF). Leukaemi and Lymphoma 1994;13:235-47.

43 Aringer M, Graninger WB, Smolen JS, Kiener HP, Steiner $\mathrm{CW}$, Trautinger F, et al. Photopheresis treatment enhances CD95 (fas) expression in circulating lymphocytes of patients with systemic sclerosis and induces apoptosis. $\mathrm{Br}$ Rheumatol 1997;36:1276-82

44 Graninger WB, Smolen JS. Should the clinician have interest in the deregulation of apoptosis in autoimmunity. Br J Rheumatol 1998;36:1244-5. 